\title{
Efecto del tipo de corte y tipo de envase en la conservación de piña [Ananas comosus (L.) Merr.] ‘Oro Miel' mínimamente procesada
}

\section{Effect of cutting and packaging type on the conservation of fresh-cut 'Golden' pineapple fruit [Ananas comosus (L.) Merr.]}

ELKIN MAURICIO BUITRAGO-DUEÑNA ${ }^{1}$

SAUL DUSSÁN-SARRIA ${ }^{1,2}$

MARÍA CAMILA RIVERA-OCHOA ${ }^{1}$

LUIS EDUARDO ORDOÑEZ-SANTOS' ${ }^{1}$

Fruto de piña, variedad Oro Miel.

Foto: S. Dussán-Sarria.

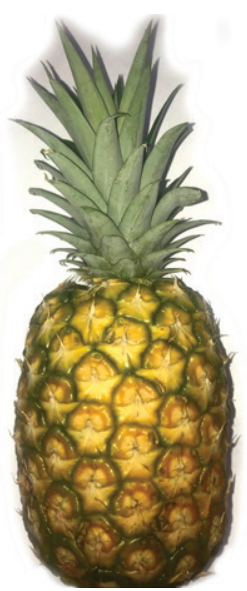

\section{RESUMEN}

La piña es un fruto tropical apreciado en el mundo por su sabor, textura y color, y es apropiado para el procesamiento mínimo. Esta investigación tuvo como objetivo determinar el efecto del tipo de corte y tipo de envase de piña mínimamente procesada sobre sus atributos de calidad durante la refrigeración a $5 \pm 1^{\circ} \mathrm{C}$ y $85-90 \%$ de humedad relativa. Durante el almacenamiento refrigerado fueron monitoreados el color, textura y aceptación sensorial durante 12 días. Las muestras se analizaron estadísticamente mediante un diseño factorial por parcelas subsubdividas completamente al azar. Los factores a evaluar fueron tres tipos de cortes y tres diferentes empaques y las variables de respuesta los atributos de calidad. Todas las mediciones fueron realizadas con tres repeticiones. Se aplicó estadística descriptiva, ANOVA y comparación de medias según Duncan. En general, tanto el tipo de corte como el tipo de empaque afectan los atributos de calidad de la piña 'Oro Miel' durante el almacenamiento. La condición más adecuada de conservación fue piña cortada en cuartos rodajas y envasada al vacío alcanzando 12 días de almacenamiento.

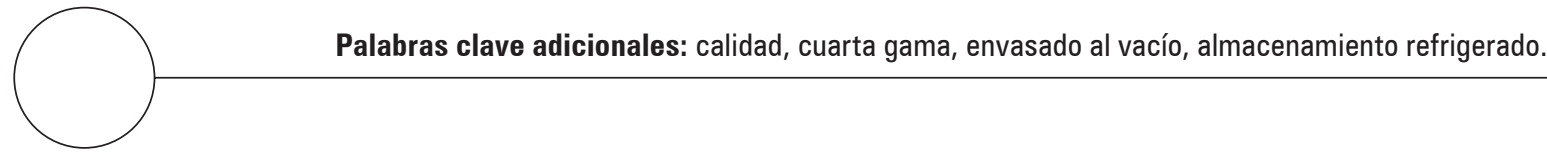

\footnotetext{
1 Facultad de Ingeniería y Administración, Universidad Nacional de Colombia, Palmira (Colombia). ORCID Buitrago-Dueñas, E.M.: 0000-0001-6950-8820; ORCID Dussán-Sarria, S.: 0000-0001-9297-0781; ORCID Rivera-Ochoa, M.C.: 0000-0002-2348-6854; ORCID Ordoñez-Santos, L.E.: 0000-0002-8958-027X

2 Autor para correspondencia.sdussan@unal.edu.co
} 


\section{ABSTRACT}

Pineapple is a tropical fruit appreciated worldwide for its flavor, texture and color, and is suitable for minimal processing. The objective of this research was to determine the effect of the cut type and type of container for minimally processed pineapple on quality attributes during refrigeration at $5 \pm 1^{\circ} \mathrm{C}$ and $85-90 \%$ relative humidity. During the refrigerated storage, the color, texture and sensorial acceptance were monitored for 12 days. The samples were analyzed statistically by means of a factorial design using completely random subsubdivided plots. The evaluated factors included three cut types and three types of packaging, and the evaluated response variables included quality attributes. All measurements were taken in triplicate. Descriptive statistics, ANOVA and means comparison using Duncan test were used. In general, both the cut type and the type of packaging affected the quality attributes of the 'Golden' pineapple during storage. The best preservation condition was pineapple cut into quarter slices and vacuum-packed, reaching 12 days of storage.

Additional key words: quality, fourth range, vacuum packaging, refrigerated storage.

Fecha de recepción: 06-11-2017 Aprobado para publicación: 30-05-2018

\section{INTRODUCCIÓN}

La piña [Ananas comosus (L.) Merr.] es una de las frutas tropicales de mayor producción en el mundo después del banano y los cítricos (Uriza-Ávila, 2005). De acuerdo con Alarcón y Sánchez (2012), la mayor producción de piña, a nivel mundial, se encuentra en Asia, América y África, lo que ha permitido el abastecimiento mundial de la fruta. En Colombia la piña es una fruta de importancia económica, se encuentra entre las 15 frutas más consumidas en el país y según cifras del DANE (2015), Colombia exportó alrededor de US\$2,25 millones en un reglón de la agricultura en crecimiento. La variedad MD2 originaria de Hawái e identificada en Colombia como piña 'Oro Miel', es la más conocida a nivel mundial, posee una forma cilíndrica, simétrica y uniforme, pericarpio entre color amarillo-naranja, pulpa de color amarillo intenso, dulce, compacta, con un peso promedio de 1,3 a 2,5 $\mathrm{kg}$, presenta un alto contenido de azúcares, vitamina C y bajo contenido de acidez en comparación con otras variedades (Chan et al., 2003). La piña mínimamente procesada cuenta actualmente con un mercado en crecimiento, ya que proveen al consumidor un alimento listo para consumir con beneficios para la salud (Escobar et al., 2014). Sin embargo, este tipo de producto tiene una vida útil de sólo $5-7 \mathrm{~d}$ a $1-7^{\circ} \mathrm{C}$, principalmente por procesos de degradación fisiológicos y microbianos (Pan et al., 2015).
Por lo tanto, si se logra inhibir la degradación fisiológica y microbiológica de la piña mínimamente procesada, es posible extender la vida útil de este tipo de productos. Dussán-Sarria et al. (2014) afirman que, si se aplican técnicas combinadas sinérgicamente, el crecimiento microbiano y las reacciones físicas, químicas y bioquímicas serian reducidas en los tejidos vegetales. En el procesamiento mínimo de piña el tipo de corte y el tipo de envase utilizado tiene una repercusión directa en la fisiología y mantenimiento de la calidad del producto vegetal. Se utilizan técnicas que se combinan sinérgicamente limitando el crecimiento microbiano y el metabolismo del producto. Algunas de estas técnicas son el enfriamiento rápido, la refrigeración, la atmosfera modificada y recubrimientos comestibles (Montero-Calderón et al., 2008; Sanjinez-Argandoña et al., 2010; Dusssán-Sarria et al., 2014). Sin embargo, la calidad de la piña mínimamente procesada variedad MD2 cultivada en Colombia, no ha sido evaluada a pesar del potencial agroindustrial que esta fruta cuenta en el suroccidente colombiano.

El objetivo de este estudio fue determinar el efecto del tipo de corte y envase apropiado para mantener la resistencia a la penetración, color y aceptación sensorial de la piña mínimamente procesada bajo condiciones de almacenamiento refrigerado. 


\section{MATERIALES Y MÉTODOS}

\section{Materia de estudio}

Los frutos de piña variedad Oro Miel, destinados para la investigación, se adquirieron en Oriente S.A., cultivados en el corregimiento Barrancas de Palmira, Valle del Cauca (temperatura $23^{\circ} \mathrm{C}, 1.000 \mathrm{msnm}, 40 \% \mathrm{HR}$ ). Luego de pruebas preliminares físico-químicas y sensoriales, se seleccionó el grado de madurez 3 (Icontec, 1996), como el adecuado para el procesamiento mínimo. Los análisis fueron realizados en el Laboratorio de Tecnología de Frutas y Hortalizas de la Universidad Nacional de Colombia, sede Palmira.

\section{Procesamiento mínimo}

Piñas enteras con grado de madurez 3 fueron lavadas con agua potable y desinfectadas en solución hipoclorito de sodio $\left(100 \mu \mathrm{L} \mathrm{L}^{-1}\right)$ durante 10 min de inmersión (Sothornvit y Rodsamran, 2008). Los frutos fueron almacenados a $10^{\circ} \mathrm{C}$ por $12 \mathrm{~h}$ previo al procesamiento. Al día siguiente, manualmente fueron retirados las coronas, epicarpio y corazones. A la pulpa se le realizaron tres tipos de corte: cuartos de rodajas $(6 \times 2 \times 4$ $\mathrm{cm})$, cubos $(2 \times 2 \times 2 \mathrm{~cm})$ y julianas $(6 \times 2 \times 2 \mathrm{~cm})$. Posteriormente los cortes fueron inmersos en solución de hipoclorito de sodio a $20 \mu \mathrm{L} \mathrm{L}^{-1}$ por 2 min para retirar impurezas en pulpa y se retiró el exceso de agua por gravedad durante 2 min. Los cortes del fruto más homogéneos fueron seleccionados y sumergidos en solución de cloruro de calcio $\mathrm{CaCl}_{2}$ al 1\% (v/v), ácido cítrico al 1\% (v/v), ácido ascórbico al 1\% (v/v) con el fin de conservar la estructura y evitar la oxidación enzimática (Robles-Sánchez et al., 2013; Dussán-Sarria et al., 2014).

Los cortes fueron sometidos a centrifugación a 2.800 rpm durante $15 \mathrm{~s}$ para eliminar el exceso de humedad. Posteriormente se acondicionaron $150 \mathrm{~g}$ de cada corte en tres tipos de empaque (Tab. 1).
Los frutos empacados fueron almacenados a $5 \pm 1^{\circ} \mathrm{C}$ y $85-90 \%$ de HR según recomienda Silva et al. (2005). Los tratamientos evaluados fueron de la siguiente manera: CR-PET: cuartos de rodaja en cajas de polietileno tereftalato (PET); C-PET: cubos en PET; J-PET: julianas en PET; CR-Poliestireno o PVC: cuartos de rodaja en bandeja de poliestireno; C-Poliestireno o PVC: cubos en bandeja de poliestireno; J-Poliestireno o PVC: julianas en bandejas de poliestireno; CR-Bolsa Vacío: cuartos de rodaja en bolsa de vacío, C-Bolsa Vacío: cubos en bolsa de vacío, J-Bolsa Vacío: julianas en bolsa de vacío. Durante el almacenamiento refrigerado, cada $3 \mathrm{~d}$ durante $12 \mathrm{~d}$ se determinaron los parámetros que se describen a continuación.

\section{Color}

El color del fruto fue evaluado mediante las coordenadas CIELab (L*, $\left.a^{*}, b^{*}\right)$, usando un colorímetro Kónica Minolta CR-410 (Tokio, Japón). De estas determinaciones y debido a las características particulares de la piña se analizó la coordenada L* que representa el brillo o luminosidad el cual aumenta a mayores valores de $\mathrm{L} \mathrm{a}^{*}$ (cromaticidad (-) verde a $(+)$ rojo) y b* (cromaticidad (-) azul a (+) amarillo).

\section{Resistencia a la penetración}

Se empleó el texturómetro Shimadzu EZ-500 (Kyoto, Japón) para evaluar la fuerza de penetración en Newton $(\mathrm{N})$ con una célula de carga de $500 \mathrm{~N}$, un cilindro de prueba de $8 \mathrm{~mm}$ de diámetro y $60 \mathrm{~mm}$ de largo, y una velocidad de ensayo de $10 \mathrm{~mm} \mathrm{~min}^{-1}$. El pico máximo durante los ensayos fue tomado como la resistencia a la penetración (Rocculi et al., 2009).

\section{Análisis sensorial}

El análisis sensorial fue realizado utilizando la metodología prueba de satisfacción de escala hedónica,

Tabla 1. Tipos de envases utilizados para el almacenamiento de piña 'Oro miel' mínimamente procesada con sus propiedades de permeabilidad.

\begin{tabular}{|c|c|}
\hline Envase utilizado & Propiedades \\
\hline $\begin{array}{l}\text { Bandeja de poliestireno cubierta con policloruro de vinilo } \\
(10 \times 10 \mathrm{~cm})\end{array}$ & PVC calibre $14 \mu \mathrm{m}$, permeabilidad al $\mathrm{CO}_{2}$ de 5.183 y al $\mathrm{O}_{2}$ de $14.803 \mathrm{~cm}^{3} \mathrm{~m}^{-2} \mathrm{~d}^{-1}$ \\
\hline $\begin{array}{l}\text { Bolsa de polietileno de baja densidad. Espesor } 70 \mu \mathrm{m} \\
\text { (PEBD, } 15 \times 20 \mathrm{~cm} \text { ) }\end{array}$ & Permeabilidad al $\mathrm{CO}_{2}$ de $112 \mathrm{~cm}^{3} \mathrm{~m}^{-2} \mathrm{~d}^{-1}$ y al $\mathrm{O}_{2}$ de $3.940 \mathrm{~cm}^{3} \mathrm{~m}^{-2} \mathrm{~d}^{-1}$ \\
\hline Cajas de polietileno tereftalato (PET) $(11 \times 9 \times 2,5 \mathrm{~cm})$ & Permeabilidad al $\mathrm{CO}_{2}$ de $112 \mathrm{~cm}^{3} \mathrm{~m}^{-2} \mathrm{~d}^{-1}$ y al $\mathrm{O}_{2}$ de $3.940 \mathrm{~cm}^{3} \mathrm{~m}^{-2} \mathrm{~d}^{-1}$ \\
\hline
\end{tabular}


siguiendo lo propuesto por Peryamm y Pilgrim (Lim, 2011). El análisis se realizó en los días 0, 10 y 12 de almacenamiento con un grupo de 30 panelistas no entrenados los cuales analizaron el sabor y la apariencia general de los cortes de fruto. Fue utilizada la escala hedónica afectiva de 5 puntos que define el grado de satisfacción así: 1 = no me gusta, $2=$ me disgusta ligeramente, $3=$ ni me gusta ni me disgusta, $4=$ me gusta ligeramente, $5=$ me gusta mucho. Los cortes se consideraron sensorialmente aceptables con notas iguales o superiores a 3 .

\section{Análisis microbiológico}

Fueron analizados las cantidades presentes o ausentes de microrganismos aerobios mesófilos según la NTC 4519 (Icontec, 2009a), coliformes totales según la NTC 4516 (Icontec, 2009b), coliformes fecales según Invima (1998a) y recuento de mohos junto a levaduras según Invima (1998b). Los análisis fueron realizados en los días 0, 10, 12 y 15 de almacenamiento.

\section{Análisis estadístico}

Las muestras se analizaron estadísticamente mediante un diseño factorial por parcelas subdividas completamente al azar. Se realizó un análisis de varianza y comparación de medias utilizando la prueba de rango múltiple de Duncan $(P \leq 0,05)$. Los datos fueron analizados usando el software estadístico $\mathrm{SAS}^{\circledR}$ v. 9.1. Los factores a evaluar fueron los tres tipos de cortes y los tres tipos de empaques.

\section{RESULTADOS Y DISCUSIÓN}

Se encontró un descenso en la aceptación sensorial para todos los tipos de cortes y empaques evaluados (Tab. 2; Figs. 1, 2, 3, 4 y 5). En general la piña en cuartos de rodaja empacada al vacío presentó las mejores notas sensoriales de aceptación durante todo el almacenamiento refrigerado. El aroma (Fig. 2) fue uno de los parámetros más afectados sensorialmente durante el almacenamiento, autores como Torri et al. (2010) afirman que la piña mínimamente procesada pierde el aroma a fruta fresca, por la disminución de compuestos como los aromáticos-alifáticos, aldehídos, cetonas y compuestos menos polares, en contraste con el incremento de los compuestos que dan aroma desagradable como los azufrados, polares, alcoholes, cetonas y terpenos a medida que avanza el almacenamiento. De igual forma sucedió con el sabor (Fig. 3), puesto que por las reacciones enzimáticas que se desarrollan en el fruto, desarrollan sabor a fermentando según el perfil de compuestos volátiles para piña cortada y almacenada bajo refrigeración como mencionan Spanier et al. (1998). La degradación de la textura (Fig. 4) se vio afectada por la pérdida de agua en la pulpa, autores como Escobar (2014), considera que la pérdida de textura en frutas y vegetales mínimamente procesados se asocia al deterioro fisiológico de peso que probablemente surge de la plasmólisis de las células vegetales y la disminución del color (Fig. 1) en los días 6 y 9 de almacenamiento (Fig. 5). La evidencia de la degradación de la textura de la pulpa se observó en la pérdida de apariencia general de la pulpa durante todo el almacenamiento (Fig. 5). Según Antoniolli et al. (2012), la piña mínimamente procesada puede conservar sus características de fruta fresca hasta el día 6 de almacenamiento, después de ahí puede desarrollar olor desagradable y sabor a fruta fermentada.

En relación con los empaques, seguramente la utilización de la caja PET no fue lo suficientemente hermética y la bandeja de icopor con PVC (tratamiento Poliestireno) presenta una alta permeabilidad a los gases, principalmente por parte del icopor, lo que significó una atmósfera modificada incompleta produciendo alteraciones sensoriales, de firmeza y color en la piña mínimamente procesada. Por su parte el vacío proporcionado por el PEBD de $70 \mu \mathrm{m}$ (tratamiento Bolsa Vacío), fue suficiente para evitar intercambios gaseosos con el producto, que produjeran alteraciones significativas durante el periodo de almacenamiento evaluado.

Considerando el empaque al vacío como el mejor aceptado sensorialmente, en valores de firmeza y color, y de acuerdo con las notas sensoriales encontradas en el atributo de aceptación general de piña cortada, se sugiere que el corte en cuartos de rodaja (CR) es el preferido por los panelistas.

Durante el procesamiento mínimo de la piña, suceden diferentes operaciones unitarias como selección, lavado, pelado, higienización, centrifugación y empaque, y el producto continúa con el proceso metabólico de maduración, favoreciendo en muchas ocasiones la proliferación de microorganismos (Rangel y López, 2012). En el día 0 de almacenamiento no hubo presencia de microorganismos tipo aerobios mesófilos, coliformes totales, coliformes fecales, ni mohos y 
Tabla 2. Valores de notas sensoriales de piña 'Oro Miel' mínimamente procesada con 30 panelistas no entrenados y una escala hedónica de 5 puntos.

\begin{tabular}{|c|c|c|c|c|}
\hline \multirow{2}{*}{ Tratamiento } & \multirow{2}{*}{ Días } & \multicolumn{3}{|c|}{ Variables de respuesta (tratamiento corte-empaque) } \\
\hline & & Efecto del aroma & Efecto del sabor & Aceptación general \\
\hline \multirow{3}{*}{ CR-PET } & 0 & $4,2 \pm 0,84$ & $4,4 \pm 0,55$ & $4,4 \pm 0,54$ \\
\hline & 10 & $2,8 \pm 0,84$ & $4,0 \pm 0,70$ & $3,6 \pm 0,54$ \\
\hline & 12 & $2,4 \pm 0,9$ & $2,8 \pm 1,30$ & $3,2 \pm 0,45$ \\
\hline \multirow{3}{*}{ C-PET } & 0 & $4,4 \pm 0,9$ & $4,6 \pm 0,55$ & $4,2 \pm 0,45$ \\
\hline & 10 & $2,8 \pm 0,45$ & $3,0 \pm 01,22$ & $3,2 \pm 0,84$ \\
\hline & 12 & $2,2 \pm 0,84$ & $2,6 \pm 0,55$ & $2,8 \pm 0,84$ \\
\hline \multirow{3}{*}{ J-PET } & 0 & $4,4 \pm 0,55$ & $4,6 \pm 0,55$ & $4,2 \pm 0,45$ \\
\hline & 10 & $2,4 \pm 1,14$ & $2,0 \pm 1,0$ & $2,0 \pm 1,0$ \\
\hline & 12 & $1,6 \pm 0,90$ & $2,0 \pm 1,00$ & $1,8 \pm 1,1$ \\
\hline \multirow{3}{*}{ CR-poliestireno } & 0 & $4,4 \pm 0,55$ & $4,2 \pm 0,45$ & $4,2 \pm 0,45$ \\
\hline & 10 & $2,4 \pm 0,9$ & $3,8 \pm 1,1$ & $3,6 \pm 0,90$ \\
\hline & 12 & $1,8 \pm 0,45$ & $2,0 \pm 1,0$ & $2,2 \pm 0,45$ \\
\hline \multirow{3}{*}{ C-poliestireno } & 0 & $4,2 \pm 0,4$ & $4,2 \pm 0,84$ & $4,0 \pm 0,70$ \\
\hline & 10 & $2,6 \pm 0,9$ & $3,0 \pm 1,58$ & $2,8 \pm 0,84$ \\
\hline & 12 & $1,6 \pm 0,55$ & $1,4 \pm 0,89$ & $1,8 \pm 0,45$ \\
\hline \multirow{3}{*}{ J-poliestireno } & 0 & $4,8 \pm 0,45$ & $4,4 \pm 0,55$ & $4,2 \pm 0,45$ \\
\hline & 10 & $2,8 \pm 0,84$ & $3,2 \pm 0,84$ & $3,2 \pm 0,84$ \\
\hline & 12 & $2,0 \pm 1,22$ & $2,0 \pm 1,0$ & $2,2 \pm 0,84$ \\
\hline \multirow{3}{*}{ CR-BolsaVacío } & 0 & $4,6 \pm 0,55$ & $4,0 \pm 0,70$ & $4,2 \pm 0,45$ \\
\hline & 10 & $3,9 \pm 1,41$ & $3,9 \pm 0,84$ & $3,6 \pm 0,55$ \\
\hline & 12 & $3,6 \pm 1,14$ & $3,6 \pm 0,55$ & $3,6 \pm 0,45$ \\
\hline \multirow{3}{*}{ C-BolsaVacío } & 0 & $4,2 \pm 0,84$ & $4,2 \pm 0,45$ & $4,2 \pm 0,45$ \\
\hline & 10 & $3,8 \pm 0,84$ & $4,0 \pm 0,70$ & $3,8 \pm 0,45$ \\
\hline & 12 & $3,6 \pm 0,55$ & $3,2 \pm 0,44$ & $3,2 \pm 0,84$ \\
\hline \multirow{3}{*}{ J-BolsaVacío } & 0 & $4,2 \pm 0,45$ & $4,2 \pm 0,45$ & $4,2 \pm 0,45$ \\
\hline & 10 & $4,0 \pm 0,70$ & $3,6 \pm 0,89$ & $3,8 \pm 0,45$ \\
\hline & 12 & $3,8 \pm 0,45$ & $3,6 \pm 0,89$ & $3,2 \pm 0,54$ \\
\hline \multicolumn{5}{|l|}{ ANOVA } \\
\hline \multicolumn{2}{|l|}{ Tiempo (T) } & $* * *$ & $* * *$ & $* * *$ \\
\hline \multicolumn{2}{|c|}{ Tratamiento corte-empaque } & NS & NS & * \\
\hline
\end{tabular}

Promedios \pm desviación estándar; $\left({ }^{*}\right)$ significativo $\left.P \leq 0,05 ;{ }^{* *}\right)$ significativo $P \leq 0,01 ;\left({ }^{* * *}\right)$ significativo $P \leq 0,001$.

CR: cuartos de rodaja; C: cubos; J: julianas; PET: cajas de polietileno tereftalato; poliestireno: bandeja de poliestireno y cubierta policloruro de vinilo; Bolsa Vacío: bolsa de polietileno de baja densidad al vacío.

levaduras. En el día 12 de almacenamiento se observó formación de colonias de algunos microorganismos, sin embargo, los recuentos fueron inferiores a los tolerables a la salud humana según la Resolución 3929 (Ministerio de Salud y Protección Social en Colombia,
2013) y De Pablo y Moragas (2008). En el día 15 de almacenamiento el recuento de todos los grupos de microorganismos cuantificados sobrepasó los umbrales aceptados por el Invima en alimentos destinados para consumo humano. 

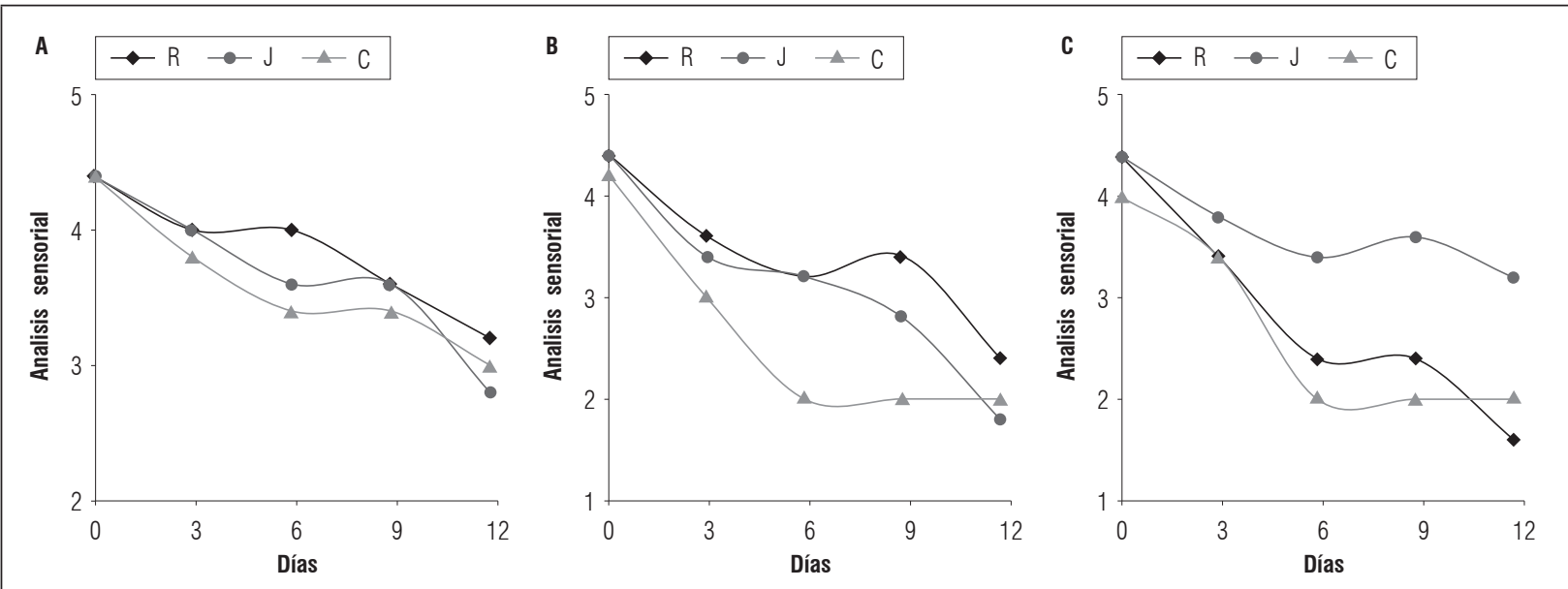

Figura 1. Evaluación de la aceptación sensorial del color en piña 'Oro Miel' mínimamente procesada con 30 panelistas no entrenados y una escala hedónica de 5 puntos. A. Envase de bolsa de vacío; B. Envase de PVC o poliestireno; C. Envase en PET; R. Cuartos de rodaja; J. Julianas; C. Cubos.

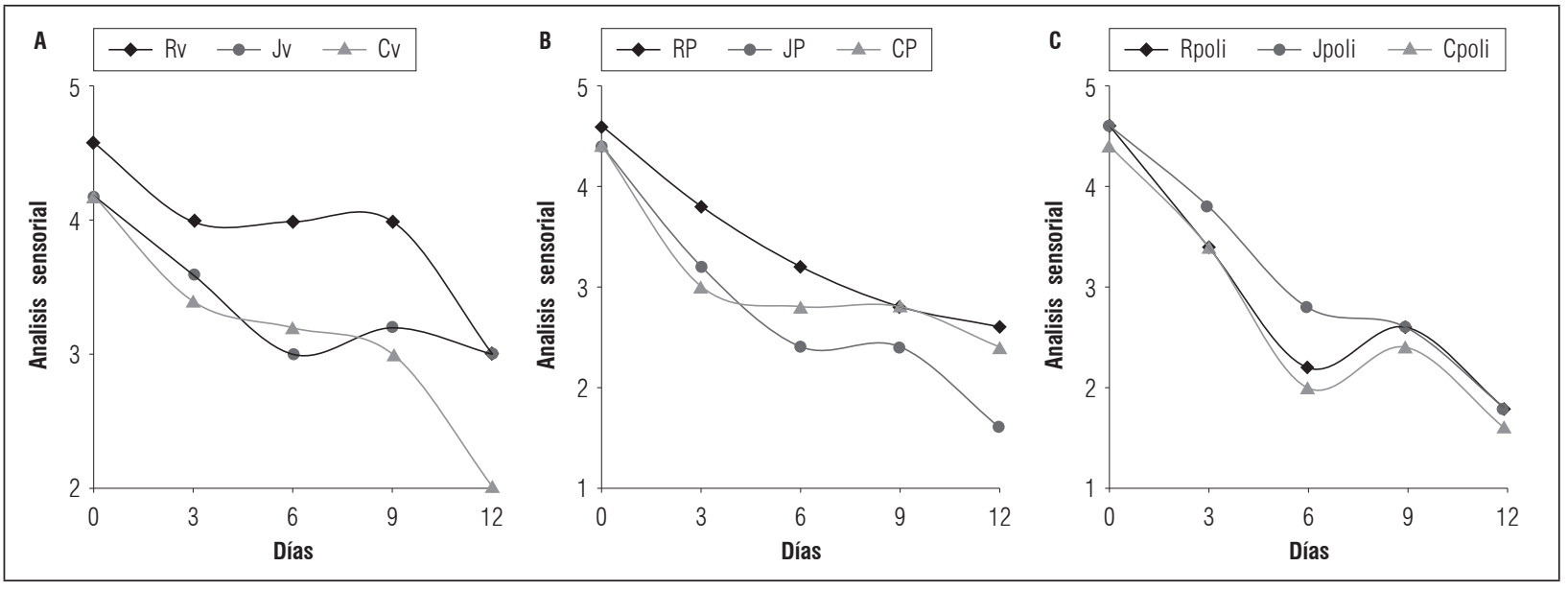

Figura 2. Evaluación de la aceptación sensorial del aroma en piña ‘Oro Miel' mínimamente procesada con 30 panelistas no entrenados y una escala hedónica de 5 puntos. A. Envase de bolsa de vacío (v); B. Envase de PVC o poliestireno (P); C. Envase en PET o polietileno tereftalato (poli); R. Cuartos de rodaja; J. Julianas; C. Cubos.

\section{Resistencia a la penetración}

La tabla 3 presenta los resultados obtenidos para firmeza, es posible observar que no existe diferencia estadística respecto al tipo de corte ni tipo de empaque. Los tratamientos con empaque al vacío exhibieron presencia de líquido exudado, sin embargo por ser un valor inferior al $1 \%$ no se consideró representativo. La pérdida de firmeza está relacionada directamente con una pérdida de agua debido a la degradación de membranas durante el almacenamiento bajo refrigeración (Portela y Cantwell, 2001; Escobar et al., 2014). Resultados similares reportan Santos et al. (2005), los autores asocian este ablandamiento, con la degradación de las pectinas asociada a la acción de las enzimas pectinametilesterasa y poligalacturonasa. De acuerdo con Toivonen y Brummell (2008), el ablandamiento de los tejidos vegetales es uno de los mayores problemas que limitan la vida útil de los frutos mínimamente procesados, y la firmeza es un factor importante que influencia la aceptabilidad del consumidor de ese tipo de productos (Rojas-Graü et al., 2008).

La pérdida de líquido exudado de productos vegetales frescos se debe a la pérdida de agua originada por el proceso de transpiración influenciado por la diferencia 

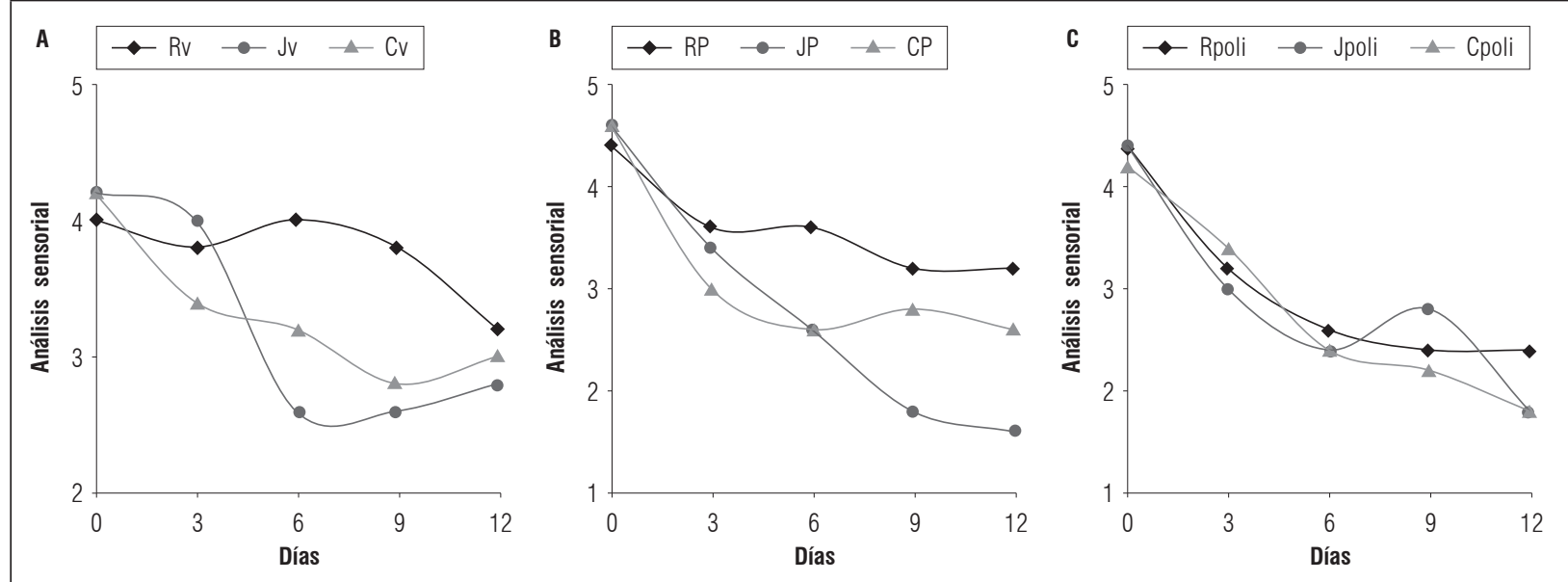

Figura 3. Evaluación de la aceptación sensorial del sabor en piña 'Oro Miel' mínimamente procesada con 30 panelistas no entrenados y una escala hedónica de 5 puntos. A. Envase de bolsa de vacío (v); B. Envase de PVC o poliestireno (P); C. Envase en PET o polietileno tereftalato (poli); R. Cuartos de rodaja, J. Julianas, C. Cubos.
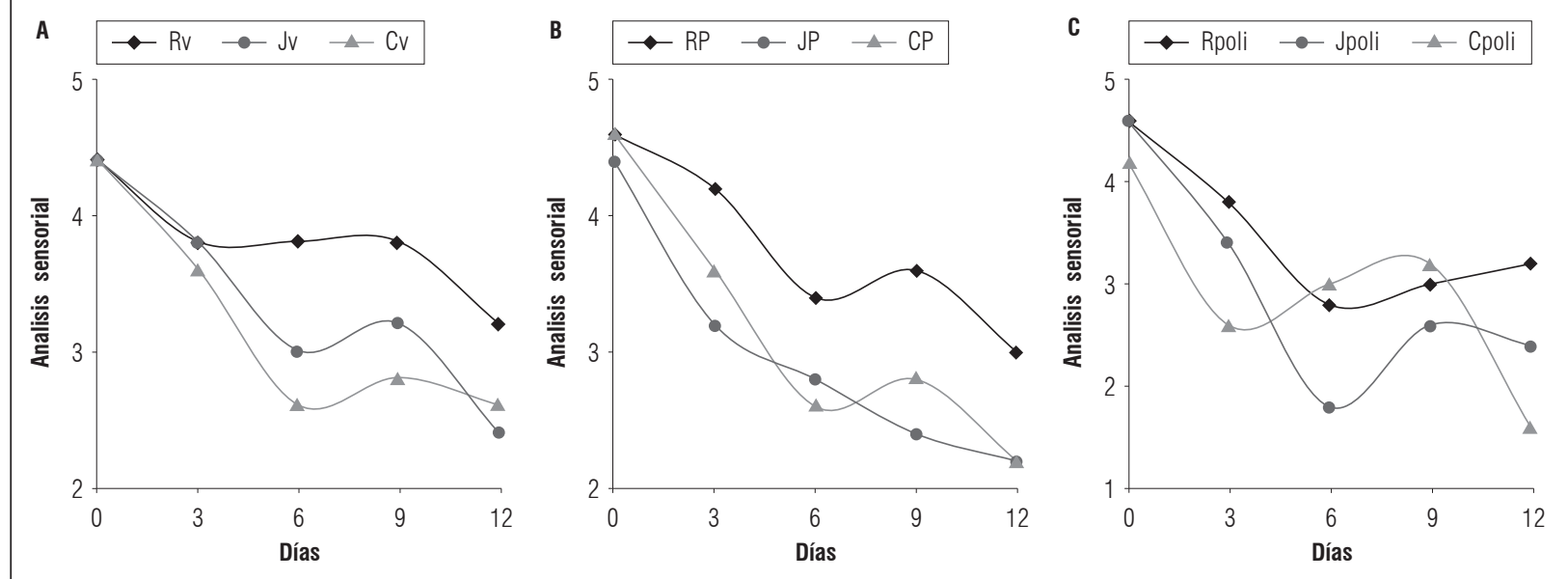

Figura 4. Evaluación de la aceptación sensorial de la textura en piña 'Oro Miel' mínimamente procesada con 30 panelistas no entrenados y una escala hedónica de 5 puntos. A. Envase de bolsa de vacío (v); B. Envase de PVC o poliestireno (P); C. Envase en PET o polietileno tereftalato (poli); R. Cuartos de rodaja; J. Julianas; C: Cubos.

de presión de vapor entre la muestra de fruto y el ambiente de refrigeración. La pérdida de líquido se hace más evidente en productos frescos cortados (Kader, 2002; Barreiro y Sandoval, 2006). Los leves cambios en los valores de los parámetros físico-químicos explican el comportamiento típico de vegetales no climatéricos como la piña (Kader, 2002).

\section{Color}

En la tabla 4 se observan los valores de L* o luminosidad. La piña en cubos presentó los mayores valores de luminosidad, seguida de la piña en julianas y por último la piña en cuartos de rodaja. La disminución de $L^{*}$ en frutas mínimamente procesadas se asocia a pardeamiento por la acción de la enzima polifenoloxidasa, pérdida gradual de agua o deshidratación superficial (Pérez et al., 2016; Djioua et al., 2010). Resultados similares reportaron Chitarra y Da Silva (1999) y Sarzi y Durigan (2002) en piña Perola, almacenada a $9^{\circ} \mathrm{C}$ durante $12 \mathrm{~d}$. El cierto grado de pérdida de luminosidad de la piña puede ser indicativo de mejor aceptación por el consumidor por asociarse a mejor sabor y suculencia (Sarzi y Durigan, 2002). 

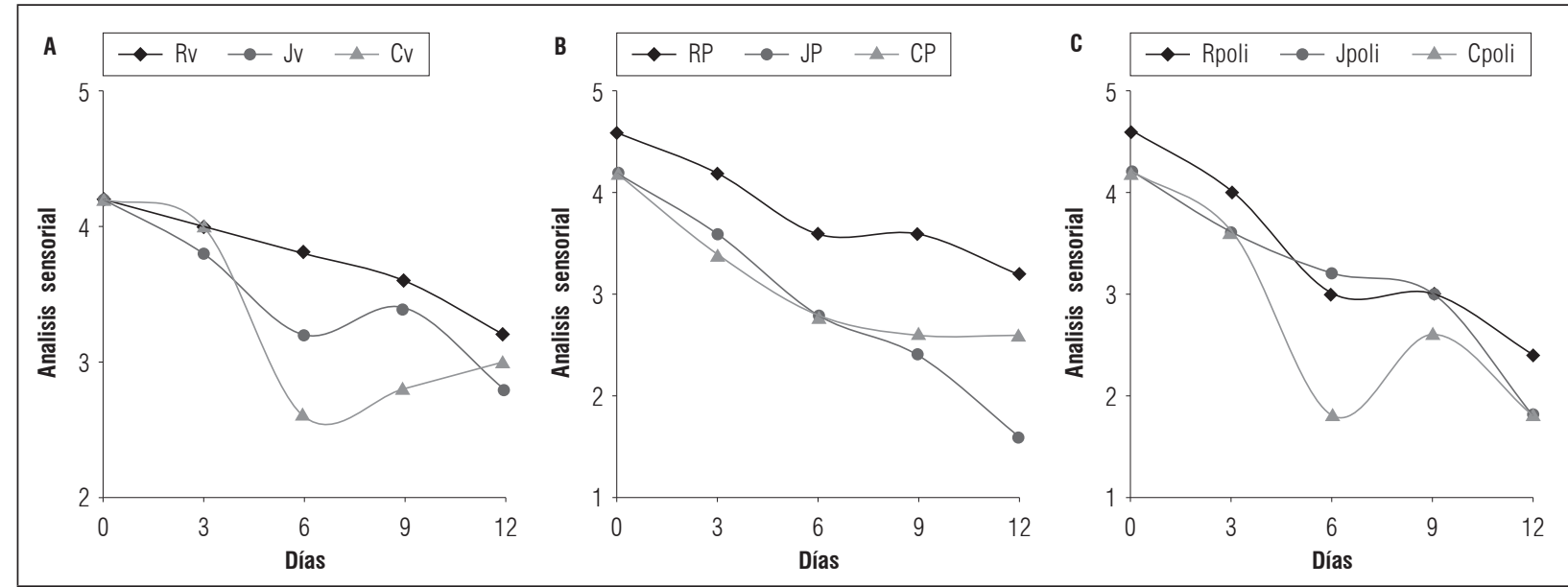

Figura 5. Evaluación de la apariencia general en piña 'Oro Miel' mínimamente procesada a través de la aceptación sensorial con 30 panelistas no entrenados y una escala hedónica de 5 puntos. A. Envase de bolsa de vacío (v); B. Envase de PVC o poliestireno (P); C. Envase en PET o polietileno tereftalato (poli); R. Cuartos de rodaja; J. Julianas; C. Cubos.

Tabla 3. Análisis comparativo del efecto del tipo de corte y envase sobre los valores de firmeza y líquido exudado de piña 'Oro Miel' mínimamente procesada para el día 12 de almacenamiento.

\begin{tabular}{|c|c|c|}
\hline Muestra & Firmeza (N) & Liquido exudado (\%) \\
\hline C-PVC & $8,38 \pm 2,0$ & $0 \pm 0$ \\
\hline C- PET & $7,48 \pm 2,1$ & $0 \pm 0$ \\
\hline C- Vacío & $6,61 \pm 2,8$ & $0 \pm 0$ \\
\hline J-PVC & $9,76 \pm 2,6$ & $0 \pm 0$ \\
\hline J-PET & $9,40 \pm 1,4$ & $0 \pm 0$ \\
\hline J- Vacío & $7,48 \pm 1,1$ & $0 \pm 0$ \\
\hline CR-PVC & $9,47 \pm 2,4$ & $0,10 \pm 2,8$ \\
\hline CR-PET & $8,69 \pm 5,3$ & $0,33 \pm 2,7$ \\
\hline CR-Vacío & $8,52 \pm 2,3$ & $0,96 \pm 2,9$ \\
\hline
\end{tabular}

Promedios \pm desviación estándar.

Promedios con letras distintas, en una misma columna, indican diferencia significativa según la prueba de rango múltiple de Duncan $(P \leq 0,05)$.

C: corte en cubos; J: corte en julianas; CR: corte en cuartos de rodajas; PVC: bandejas de poliestireno cubierta con policloruro de vinilo; PET: cajas de polietileno tereftalato; Vacío: bolsa de polietileno de baja densidad al vacío.

Para la coordenada cromática $a^{*}$ que se ubicó entre los valores negativos que indican verde, no presentó diferencias significativas $(P \leq 0,05)$ entre los envases pero sí entre los cortes. Puede estar asociada a las diferencias naturales del color dentro de un mismo fruto (Sarzi y Durigan, 2002).

Montero-Calderón et al. (2008) observaron igualmente una reducción del $22 \%$ en los valores de la coordenada L* en piña mínimamente procesada con
Tabla 4. Análisis comparativo del efecto del tipo de corte y envase sobre el color en piña 'Oro Miel' mínimamente procesada para el día 12 de almacenamiento.

\begin{tabular}{|c|c|c|c|}
\hline Muestra & $\mathrm{L}$ & $\mathrm{a}^{*}$ & $\mathrm{~b}^{*}$ \\
\hline C-PVC & $60,06 \pm 4,1 \mathrm{ac}$ & $-2,05 \pm 1,2 \mathrm{a}$ & $29,93 \pm 2,8 \mathrm{c}$ \\
\hline C- PET & $61,32 \pm 4,3 \mathrm{bc}$ & $-2,25 \pm 0,9 \mathrm{a}$ & $29,61 \pm 2,1 \mathrm{cb}$ \\
\hline C- Vacío & $57,22 \pm 4,4 \mathrm{c}$ & $-1,85 \pm 0,6 \mathrm{a}$ & $30,17 \pm 2,3 \mathrm{cb}$ \\
\hline J-PVC & $57,60 \pm 6,1 \mathrm{ab}$ & $-1,71 \pm 1,7 \mathrm{~b}$ & $29,09 \pm 1,7 \mathrm{~b}$ \\
\hline J-PET & $59,61 \pm 5,4 \mathrm{~b}$ & $-1,94 \pm 0,7 \mathrm{~b}$ & $27,09 \pm 2,5 \mathrm{bc}$ \\
\hline J- Vacío & $55,17 \pm 3,5 \mathrm{c}$ & $-1,43 \pm 0,7 \mathrm{~b}$ & $28,11 \pm 2,7 \mathrm{~b}$ \\
\hline CR-PVC & $47,72 \pm 3,1 \mathrm{ab}$ & $-3,13 \pm 1 \mathrm{c}$ & $26,08 \pm 3,1 \mathrm{ba}$ \\
\hline CR-PET & $46,42 \pm 3,2 \mathrm{ac}$ & $-2,91 \pm 0,9 \mathrm{c}$ & $24,30 \pm 2,2 \mathrm{a}$ \\
\hline CR-Vacío & $49,18 \pm 2,9 \mathrm{ca}$ & $-3,53 \pm 0,6 \mathrm{c}$ & $27,52 \pm 2,1 \mathrm{cb}$ \\
\hline
\end{tabular}

Promedios \pm desviación estándar.

Promedios con letras distintas, en una misma columna, indican diferencia significativa según la prueba de rango múltiple de Duncan $(P \leq 0,05)$.

C: corte en cubos; J: corte en julianas; R: corte en cuartos de rodajas; PVC: bandejas de poliestireno cubierta con policloruro de vinilo; PET: cajas de polietileno tereftalato; Vacío: bolsa de polietileno de baja densidad al vacío.

recubrimiento comestible de alginato de sodio y adición de cloruro de calcio a 20 d de almacenamiento a $5^{\circ} \mathrm{C}$. González-Aguilar et al. (2004) también observaron una disminución significativa de las coordenadas de color L* durante $14 \mathrm{~d}$ de almacenamiento de rodajas de piña tratadas con ácido ascórbico (0,05\%).

De acuerdo con Antoniolli et al. (2003), el cloruro de calcio aplicado en trozos de piña resultó en descenso de los valores de la coordenada $L^{*}$ indicando cierto 
oscurecimiento de las muestras. Por otro lado, Meléndez-Martínez, et al. (2004) afirman que la adición de antioxidantes, evita la disminución de la luminosidad de piña durante el procesamiento y almacenamiento.

La disminución de los valores de la coordenada b* corresponden a un cambio de tonalidad amarillo a una tonalidad más oscura en la piña mínimamente procesada, esto debido a la degradación de carotenoides asociados a la coloración amarilla. Considerando un mismo tipo de corte, la piña acondicionada al vacío exhibió los mayores valores de b*, esto se atribuye a la exclusión del oxígeno, contribuyendo a que los carotenoides no presentaran degradación alguna y por tal motivo se mantuviera el color característico de la piña.

\section{CONCLUSIONES}

La piña 'Oro Miel', mínimamente procesada, presenta alteraciones físico-químicas y cambios sensoriales durante el almacenamiento refrigerado por efecto del tipo de corte y tipo de empaque.

De acuerdo a las características de calidad evaluadas para cada tipo de corte y empaque y la evaluación sensorial, es posible establecer que la piña variedad Oro Miel mínimamente procesada, es aceptable para el consumidor hasta el día 12 de almacenamiento.

El tipo de corte y empaque que conserva mejor las características de calidad de la piña Oro Miel mínimamente procesada y que presentó mejor aceptabilidad, corresponde al corte en cuartos de rodajas empacado al vacío.

Conflicto de intereses: el manuscrito fue preparado y revisado con la participación de los autores, quienes declaran no tener algún conflicto de interés que coloquen en riesgo la validez de los resultados aquí presentados.

\section{REFERENCIAS BIBLIOGRÁFICAS}

Alarcón M. y P. Sánchez 2012. Estudio de factibilidad para la implementacion de uma fabrica dedicada a la elaboracion de jugos de piña que ayude a rescatar el cultivo de esta fruta en la zona del Milagro. Trabajo de grado. Facultad Ciencias Administrativas y Comerciales, Universidad Estatal de Milagro, San Francisco de Milagro, Ecuador.
Antoniolli, L., B.C. Benedito y S.M. Sousa Filho. 2003. Efeito do cloreto de cálcio na qualidade de abacaxi" Pérola" minimamente procesado. Pesq. Agrop. Bras. 38(9), 1105-1110. Doi: 10.1590/S0100-204X2003000900012

Barreiro, J. y A. Sandoval. 2006. Operaciones de conservación de alimentos por bajas temperaturas. Universidad Simon Bolívar; Editorial Equinoccio, Caracas, Venezuela.

Chan, Y.K., G.C. d'Eeckenbrugge y G.M. Sanewski. 2003. Breeding and variety improvement. pp. 3356. En: Bartholomew, D.P., R.E. Paull, y K.G. Rohrbach (eds.). The pineapple: botany, production and uses. CABI Publishing, Wallingford, UK. Doi: 10.1079/9780851995038.0033

Chitarra, A.B. y J.M. Da Silva. 1999. Effect of modified atmosphere on internal browning of 'Smooth Cayenne' pineapples. Acta Hortic. 485, 85-90. Doi: 10.17660/ ActaHortic.1999.485.10

DANE (Departamento Administrativo Nacional de Estadística de Colombia). 2015. Boletín técnico de exportaciones. Bogotá, Colombia.

De Pablo, B. y M. Moragas. 2008. Recopilación de normas microbiológicas de los alimentos y asimilados y otros parámetros físico-químicos de interés sanitario. En: http://www.eurocarne.com/daal?a1=informes\&a2 =normas-microbiologicas.pdf; consulta: abril de 2017

Djioua, T., F. Charles, F. Lopez-Laun, H. Filgueiras, A. Coudret, J. Freire, M. Ducamp-Collin y H. Sallanon. 2010. Improving the storage of minimally processed mangoes (Mangifera indica L.) by hot water treatments. Postharvest Biol. Technol. 52(2), 221-226. Doi: 10.1016/j.postharvbio.2008.10.006

Dussán-Sarria, S., P.M. Reyes-Calvache y J.I. Hleap-Zapata. 2014. Efecto de un recubrimiento comestible y diferentes tipos de empaque en los atributos físico-químicos y sensoriales de piña 'manzana' mínimamente procesada. Inf. Tecnol. 25(5), 41-46. Doi: 10.4067/S0718-07642014000500007

Escobar, A., C. Márquez., C. Restrepo y L. Pérez. 2014. Aplicación de tecnología de barreras para la conservación de mezclas de vegetales mínimamente procesados. Rev. Fac. Nac. Agron. Medellín 67(1), 7238-7245. Doi: 10.15446/rfnam.v67n1.42652

González-Aguilar, G.A., S. Ruiz-Cruz, R. Cruz-Valenzuela, A. Rodriguez-Félix y C.Y. Wang. 2004. Physiological and quality changes of fresh-cut pineapple treated with antibrowning agents. Lebensm.-Wiss. Technol. 37(3), 369-376. Doi: 10.1016/j.lwt.2003.10.007

Icontec (Instituto Colombiano de Normas Técnicas y Certificación). 1996. Norma Técnica Colombiana NTC 729-1. Frutas frescas. Piña. Especificaciones. Bogotá, Colombia 
Icontec (Instituto Colombiano de Normas Técnicas y Certificación). 2009a. Norma Técnica Colombiana NTC 4519. Microbiología de los alimentos para consumo humano y animal. Método horizontal para el recuento de microorganismos. Técnica de recuento de colonias a $30^{\circ} \mathrm{C}$. Bogotá, Colombia.

Icontec (Instituto Colombiano de Normas Técnicas y Certificación). 2009b. Norma Técnica Colombiana NTC 4516. Microbiología de alimentos y productos de alimentación animal. Método horizontal para la detección y enumeración de coliformes técnica del número más probable. Bogotá, Colombia.

Invima (Instituto Nacional de Vigilancia de Medicamentos y Alimentos). 1998a. Manual de técnicas para el análisis de Alimentos: método No. 7. Bogotá, Colombia.

Invima (Instituto Nacional de Vigilancia de Medicamentos y Alimentos). 1998b. Manual de Técnicas para el análisis de Alimentos: método No. 14. Bogotá, Colombia.

Kader, A.A. 2002. Post-harvest technology of horticultural crops. Division of Agriculture and Natural Resources Publication No. 3311, University of California, Oakland, CA, USA.

Lim, J. 2011. Hedonic scaling: a review of methods and theory. Food Qual. Prefer. 22, 733-747. Doi: 10.1016/j. foodqual.2011.05.008

Meléndez-Martínez, A.J., I.M. Vicario y F.J. Heredia. 2004. Estabilidad de los pigmentos carotenoides en los alimentos. Arch. Latinoam. Nutr. 54(2), 209-215.

Ministerio de Salud y Protección Social, Republica de Colombia. 2013. Resolución 3929, reglamento técnico sobre los requisitos sanitarios que deben cumplir las frutas y las bebidas con adición de jugo (zumo) o pulpa de fruta o concentrados de fruta, clarificados o no, o la mezcla de estos que se procesen, empaquen, transporten, importen y comercialicen en el territorio nacional. Diario Oficial No. 48.933. Bogotá, Colombia.

Montero-Calderón, M., M.A. Rojas-Graü y O. Martín-Belloso. 2008. Effect of packaging on quality and shelf-life of fresh cut-pineapple (Ananas comosus). Postharvest Biol.Technol. 50, 182-189. Doi: 10.1016/j. postharvbio.2008.03.014

Pan, Y., J. Zhu y S. Li. 2015. Effects of pure oxygen and reduced oxygen modified atmosphere packaging on the quality and microbial characteristics of freshcut pineapple. Fruits 70(2), 101-108. Doi: 10.1051/ fruits/2015003

Pérez, A.F., I.D. Aristizábal y J.I. Restrepo. 2016. Conservación de mango Tommy Atkins mínimamente procesado mediante la aplicación de un recubrimiento de aloe vera (Aloe barbandensis Miller). Vitae 23(1), 65-77. Doi: 10.17533/udea.vitae.v23n1a02
Portela, S. y M. Cantwell. 2001. Cutting blade sharpness affects appearance and other quality attributes of fresh-cut cantaloupe melon. J. Food Sci. 66(9), 12651270. Doi: 10.1111/j.1365-2621.2001.tb15199.x

Rangel, M. y A. López. 2012. Cambios en frutas tropicales frescas, cortadas y empacadas en atmósfera modificada durante su almacenamiento en refrigeración. Temas Select. Ing. Aliment. 6(2), 94-109.

Robles-Sánchez, R., A. Rojas-Graü, I. Odriozola-Serrano, G. González-Aguila y O. Martín-Belloso. 2013. Influence of alginate-based edible coating as carrier of antibrowning agents on bioactive compounds and antioxidant activity in fresh-cut Kent mangoes. LWT-Food Sci. Technol. 50(1), 240-246. Doi: 10.1016/j.lwt.2012.05.021

Rocculi, R., E. Cocci, S. Romani, G. Sacchetti y M. Dalla Rosa. 2009. Effect of 1-MCP treatment and $\mathrm{N}_{2} \mathrm{O}$ MAP on physiological and quality changes of fresh-cut pineapple. Postharvest Biol. Technol. 51, 371-377. Doi: 10.1016/j.postharvbio.2008.07.010

Rojas-Graü, M.A., M.S. Tapia y O. Martín-Belloso, 2008. Using polysaccharide-based edible coatings to maintain quality of fresh-cut Fuji apples. Food Sci. Technol. 41, 139-147. Doi: 10.1016/j.lwt.2007.01.009

Sanjinez-Argandoña, E.J., I.G. Branco, S. Takito y J. Corbari. 2010. Influencia de la deshidratación osmótica y de la adición de cloruro de calcio en la conservación de kiwis minimamente procesados. Food Sci. Technol. 30(suppl. 1), 205-209. Doi: 10.1590/S0101-20612010000500031

Santos, J., E. Valeiro, M. Torre y A. Marquez. 2005. Avaliação da qualidade do Abacaxi 'Pérola' minimamente processado armazenado sob atmosfera modificada. Ciênc. Agrotec. 29(2), 353-361. Doi: 10.1590/ S1413-70542005000200012

Sarzi, B. y J. Durigan. 2002. Avaliação física e química de produtos minimamente processados de abacaxi-'pérola'. Rev. Bras. Frutic. 24(2), 333-337. Doi: 10.1590/ S0100-29452002000200012

Silva, G.C., G.A. Maia, R. Figueiredo, M. Souza Filho, R. Alves y M. Souza Neto. 2005. Efeito do tipo de corte nas características físico-químicas do abacaxi pérola minimamente processado. Food Sci. Technol. 25(2), 223-228. Doi: 10.1590/S0101-20612005000200006

Sothornvit, R. y P. Rodsamran. 2008. Effect of a mango film on quality of whole and minimally processed mangoes. Postharvest Biol. Technol. 47(3), 407-415. Doi: 10.1016/j.postharvbio.2007.08.005

Spanier, A., M. Flores, C. James, J. Lasater, S. Lloyd y J. Miller. 1998. Fresh-cut pineapple (Ananas sp.) flavor. Effect of storage. Dev. Food Sci. 40, 331-343. Doi: 10.1016/S0167-4501(98)80057-5

Toivonen, P.M.A. y D.A. Brummell. 2008. Biochemical bases of appearance and texture changes in fresh-cut 
fruit and vegetables. Postharvest Biol. Technol. 48, 1-14. Doi: 10.1016/j.postharvbio.2007.09.004

Torri, L., N. Sinelli y S. Limbo. 2010. Shelf life evaluation of fresh-cut pineapple by using an electronic nose. Post- harvest Biol. Technol. 56(3), 239-245. Doi: 10.1016/j. postharvbio.2010.01.012

Uriza-Ávila, D. 2005. Foreword and preface. Acta. Hortic. 666. Doi: 10.17660/ActaHortic.2005.666.0 